This is a self-archived version of an original article.

This reprint may differ from the original in pagination and typographic detail.

Title: Design and Implementation of a Flexible Interface for TID Detector

Author(s): Iacopo Fara, Lucas Matana Luza, Jérôme Boch, Gianluca Furano, Marco Ottavi, and Luigi Dilillo

DOI: 10.1109/IWASI.2019.8791299

Published: 08 August 2019

Document version: Post-print version (Final draft)

Please cite the original version:

I. Fara, L. M. Luza, J. Boch, G. Furano, M. Ottavi and L. Dilillo, "Design and Implementation of a Flexible Interface for TID Detector," 2019 IEEE 8th International Workshop on Advances in Sensors and Interfaces (IWASI), 2019, pp. 158-162, doi: 10.1109/IWASI.2019.8791299.

This material is protected by copyright and other intellectual property rights, and duplication or sale of all or part of any of the repository collections is not permitted, except that material may be duplicated by you for your research use or educational purposes in electronic or print form. You must obtain permission for any other use. Electronic or print copies may not be offered, whether for sale or otherwise to anyone who is not an authorised user. 


\title{
Design and Implementation of a Flexible Interface for TID Detector
}

\author{
$1^{\text {st }}$ lacopo Fara \\ Universitá degli Studi di Roma "Tor Vergata" \\ LIRMM \\ Rome, Italy \\ iacopo.fara@alumni.uniroma2.eu
}

\author{
$2^{\text {nd }}$ Lucas Matana Luza \\ LIRMM \\ Université de Montpellier / CNRS \\ Montpellier, France \\ lucas.matana-luza@lirmm.fr
}

\author{
$3^{\text {rd Jérôme Boch }}$ \\ IES
}

Université de Montpellier

Montpellier, France

jerome.boch@umontpellier.fr

\author{
$4^{\text {th }}$ Gianluca Furano \\ ESA / ESTEC \\ Noordwijk, Netherlands \\ Gianluca.Furano@esa.int
}

\author{
$6^{\text {th }}$ Luigi Dilillo \\ LIRMM \\ Université de Montpellier / CNRS \\ Montpellier, France \\ dilillo@lirmm.fr
}

\begin{abstract}
This paper introduces a flexible sensor interface for a radiation Total lonizing Dose (TID) monitor. The board is conceived to be flexible and could be used with several detectors, the results reported in this work are based on the radiation-induced shift of the threshold voltage of a FET transistor that is polarized by current. The system has been tested for a range of radiation in Co60 facility up to about $5 \mathrm{krad}$, which corresponds to the double of the TID that will face the Cubesat MTCUBE for a two-year nominal mission in a low orbit.
\end{abstract}

\section{Index Terms}

Radiation, TID, sensor, RADFET, data acquirement

\section{INTRODUCTION}

In this work, we deal with electronic components that work in radiation environments. Radiation environments can be split into two main categories: natural environments such as space, or artificial as it is present in particle accelerators or nuclear reactors. The space radiation environment can be divided into three main groups: the particles originating from the Sun; the Galactic Cosmic Rays (GCRs); and the trapped particles in the Earth's magnetic field [1].

Radiations affect electronic devices in multiple ways: particles (such as photons, electrons, neutrons or heavy ions) that impact the devices can lead to irreversible (partially or totally) or reversible faults, as exposed in [2], with Single Event Effects (SEEs) or cumulative effects (Total lonizing Dose, Displacement Damage).

SEEs can lead to unexpected behavioural of the involved electronics circuits, as in memories [3]. In Displacement Damages, incident radiation can change the crystalline structure of the material causing an alteration of the material's properties, and these problems afflict more the devices based on bulk conduction (e.g., BJTs, diodes, JFETs) [4].

Total dose effects are generated when impinging particles create an excess charge in the dielectric layers used for insulation in electronic devices of an integrated circuit, as MOSFET; a model is given in [5]. For integrated circuits (ICs), extended exposure to TID radiation can shift the threshold voltages, making transistors easier or harder to switch. Radiation may also increase the leakage current, causing ON and OFF states of the transistors to become less distinguishable, other effects can lead to circuit failure. For these reasons, it is essential to have instruments capable of measuring TID. The physical mechanism of components able to measuring radiation are exposed in [6].

This work introduces an electronic interface of a TID sensor based on the measure of voltage threshold shifting of four Field Effect Transistors (FET). The test setup and interface are discussed in the following sections.

The main contribution of this paper is the design and implementation on a flexible interface for TID detectors, thus providing a multipurpose platform for TID detection.

The paper is organized as follows: Section II explains in detail the TID sensor and its setup and interface; in Section III there is the system description and the procedure for data acquisition; Section IV provides the preliminary experimental data for TID sensor; finally, Section V provides the work conclusion. 


\section{TID SENSOR DESCRIPTION}

The board developed in this work is amenable for interface with different kinds of TID detectors, in particular also with digitally controlled radiation sensor with digital output such as FGDOS [7]. The DUT (Device Under Test) that is considered in the following belongs to the category of RADFET (RADiation sensitive Field Effect Transistor) with an analog readout interface.

RADFETs are integrating ionizing radiation dosimeters which operate on the principle of converting the radiationinduced threshold voltage shift into absorbed dose. RADFETs measure the dose (or more precisely the ionization energy) deposited in the Silicon gate oxide layer of the FET, through generation and trapping of radiation-induced charges.

In our accelerated experiments the TID RADFET is irradiated and, through the acquisition board, the variation of its threshold voltage is measured. The RADFET used for this work is a p-channel MOSFET which is sensitive to ionizing radiation: protons, $\mathrm{X}$-rays and gamma rays.

The target DUT is manufactured by Tyndall National Institute, and it has been tested under radiation at the $\mathrm{Co}_{60}$ facility of the University of Montpellier. A photo of the used component on a daughter board is shown in Fig. 1.

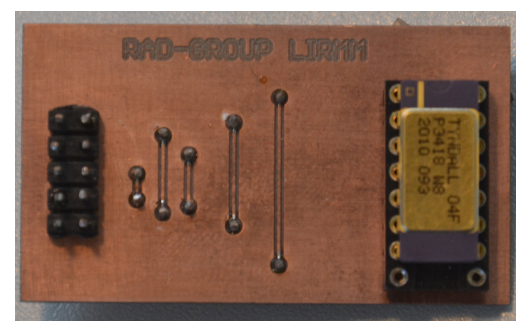

Fig. 1. Photo of the Tyndall RADFET on its support board.

The Tyndall RADFET chip TY1001 consists of four p-channel MOSFETs/RADFETs and a diode (as presented in Fig. 2 [8]). The RADFET gate oxide thickness is $400 \mathrm{~nm}$, and W/L of the two isolated FETs is $300 \mu \mathrm{m} / 50 \mu \mathrm{m}$ and $690 \mu \mathrm{m} / 15 \mu \mathrm{m}$ meanwhile the other two FETs are one of one type and one of another, equal to the previous ones. The former two RADFETs have individual gate and drain terminals, while the source and bulk are common and connected; this is also the diode bulk contact. The last two RADFETs have a common drain, and gate terminals with the source and bulk are linked to the former ones. Biasing each FET is possible by using switches on the acquisition board, as presented in section III.

The modes of operation of this component are substantially two:

- Irradiation Mode (Sense Mode): In this mode of operation it is recommended that all terminals of the RADFET are connected to ground and the component can be irradiated;

- Read-Out Mode (Accumulated Radiation Dose Read-Out): The RADFET can be read at arbitrary intervals, the reading is done through the acquisition board. The period between readings can be from seconds to days. The Reader Circuit (RC), which is used to read out the RADFET, is shown in Fig. 3. Current RC_I is forced into the RADFET, connected in RC configuration, for several hundred $\mathrm{ms}$. The voltage at the source (RC_V or $\left.\mathrm{V}_{\mathrm{Gs}}(\mathrm{th})\right)$ is measured by the acquisition board; this voltage is called "RC threshold voltage". The read-out current value (RC_I) can be chosen by the pump current on the acquisition board, which guarantees the stability of this current during the measurement, after the beginning of irradiation. For best temperature compensation an RC_I value of $10 \mu \mathrm{A}$ is set.

\section{SYSTEM DESCRIPTION}

The proposed sensor interface is an acquisition system that consists of: a $\Delta \Sigma$ analog-to-digital converter (ADC); a controller unit; an instrumentation amplifier; two adjustable currents sources; and three single-pole/single-throw (SPST) switches.

The system is based on the Texas Instruments Analog Front End (AFE) ADS114S08, which as a $\Delta \Sigma$-ADC with 16 bits of resolution and provide 12 configurable analog inputs (single-ended or differential) that are connected to the internal analog input $\mathrm{ANI}_{\mathrm{P}}$ or $\mathrm{ANI}_{\mathrm{N}}$ through an analog multiplexer. Also, the AFE has a low-drift, low-noise, high impedance programmable amplifier (PGA) with gains from 1 to 128 and the sampling rate can be from 2.5 up to 4,000 samples per second (SPS). The configuration of the AFE and the acquirement of the converted data is done using a SPI interface [9]. 


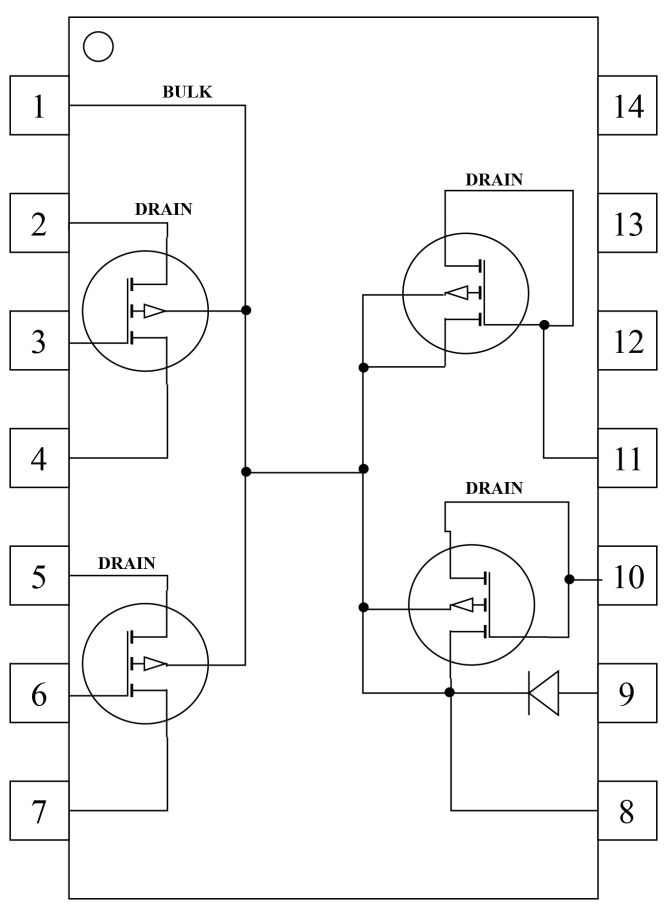

Fig. 2. Tyndall RADFET functional diagram.

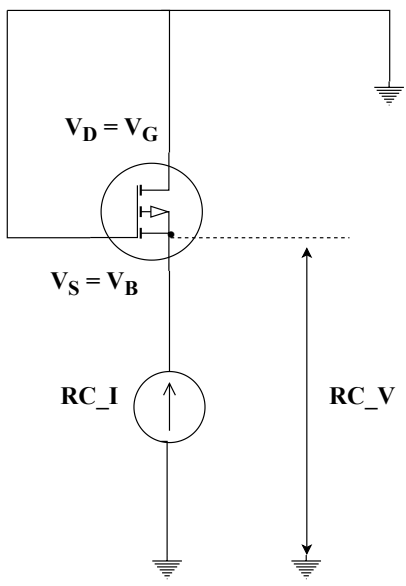

Fig. 3. Tyndall Reader Circuit (RC) configuration.

The board uses an Arduino Nano to perform the control of the AFE and also to create a user interface, being capable of storing the acquired data in a log file using a serial interface (UART) between the controller unit and a computer.

In comparative terms, considering a full scale of $5 \mathrm{~V}$, an application directly involving the ADC provided by the ATmega328 microcontroller used by the Arduino Nano, has a resolution of $4.88 \mu \mathrm{V}$, since this microcontroller has a 10 bits ADC. On the other hand, the AFE, which uses the most significant bit for the two's complement format, has a resolution of $152 \mu \mathrm{V}$.

The ATmega328 is functionally equivalent to the ATmegaS128, ATMEL released the ATmegaS128 as the space market version of the popular Atmel AVR ATmega128, similar to the one powering the Arduino boards. The space qualified version of this part adds: ceramic, hermetic package, extended temperature range $-55^{\circ} \mathrm{C}-125^{\circ} \mathrm{C}$, extended qualification flow according to QML-V or QML-Q space grade flow. Radiations performances for the ATmegaS128 are: SEL LET $>62.5 \mathrm{MeV} . \mathrm{cm}^{2} / \mathrm{mg}$, SEU LET $>3 \mathrm{MeV} . \mathrm{cm}^{2} / \mathrm{mg}$ and TID up to $30 \mathrm{krad}(\mathrm{Si})$. Several other microcontrollers are planned or in development by the major players in Hi-Rel electronics, but also use of COTS components is a valid alternative for some applications once a proper radiation characterization is performed. 
When the system is powered, the control unit starts the process configuring the communications interfaces. The $\mathrm{SPI}$ interface is set with a frequency of $1 \mathrm{MHz}$, clock polarity (CPOL) ' 0 ' and a clock phase (CPHA) ' 1 '. The UART interface is configured with a baud rate of $115,200 \mathrm{bps}$. The power-on reset is applied on the AFE to ensure that the device starts operating in a known state in case a brown-out event occurs when the supplies have dropped below the minimum operating voltage [9]. The ADS registers are used to configure and control the device to the desired operation mode. The main functions that can be configured in the system are: select the multiplexer positive and negative inputs; set the PGA gain; configure the output data rate; select the reference input; and define a single-shot or continuous read mode.

After the configuration, the system remains to wait for a user command. It is possible to request data acquisitions indicating a value of $n$ samples or enable/disable a specific switch. When an acquisition data is requested, the control unit acquires $n$ samples from the AFE and transmits the read value using the UART interface. The host computer receives the acquired data and saves them together with a timestamp in a log file. Fig. 4 presents a simplified diagram with the steps performed by the controller unit.

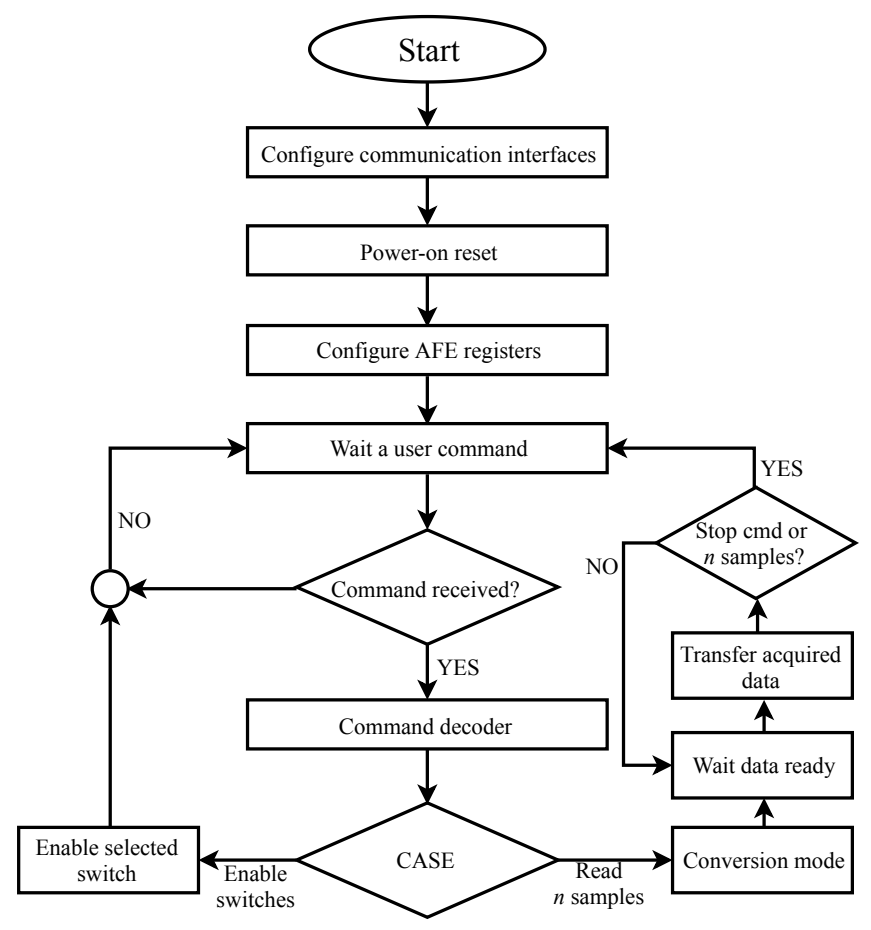

Fig. 4. Firmware diagram.

The current source circuit uses the Texas Instruments adjustable current source LM334 since it is able to follow the requirements for the Tyndall RADFET operation. This current source can be adjustable from $1 \mu \mathrm{A}$ to $10 \mathrm{~mA}$ and operates in a wide voltage range from $1 \mathrm{~V}$ to $40 \mathrm{~V}[10]$. The source current can be adjustable changing the value of two trimmers that compose the pump current circuit, the value respect the Eq. 1 and 2.

$$
\begin{gathered}
I_{\mathrm{SET}}=\frac{0.134}{R_{1}} \\
R_{2}=10 R_{1}
\end{gathered}
$$

Targeting the data acquisition from the RADFET, the system applies a circuit containing three SPST switches ADG1401, which as a $1 \Omega$ resistance and supports up to $430 \mathrm{~mA}$ continuous current [11]. This circuit allows the separate measurement of the four transistors present in the RADFET IC. The activation of the 'SW1' switch allows the current flow in the first of the IC transistors, as well as 'SW2' to the second transistor and 'SW3' to the third and forty transistors which are connected with common drain and gate.

In order to extend the acquired voltage range $\left(\mathrm{V}_{\mathrm{GS}}(\mathrm{th}) \mathrm{span}\right)$, the system provides the instrumentation amplifier of the Texas Instruments INA128, which can operate with the analog input voltage in a range of $\pm 40 \mathrm{~V}$ and has the following characteristics: low offset voltage (50 $\mu \mathrm{V}$ maximum); low input bias current (5 nA maximum); and a high 
CMR (120 dB minimum) [12]. The INA128 is configured with a ground reference. The positive input is from the DUT, and the negative input receives an external reference voltage near the pre-irradiation $\mathrm{Vgs}(\mathrm{th})$ value. The output must be between $0 \mathrm{~V}$ and $5 \mathrm{~V}$, respecting the maximum ratings of the AFE.

Fig. 5 presents the system block diagram, and Fig. 6 presents the manufactured board which has a $7 \times 9.3 \mathrm{~cm}$.

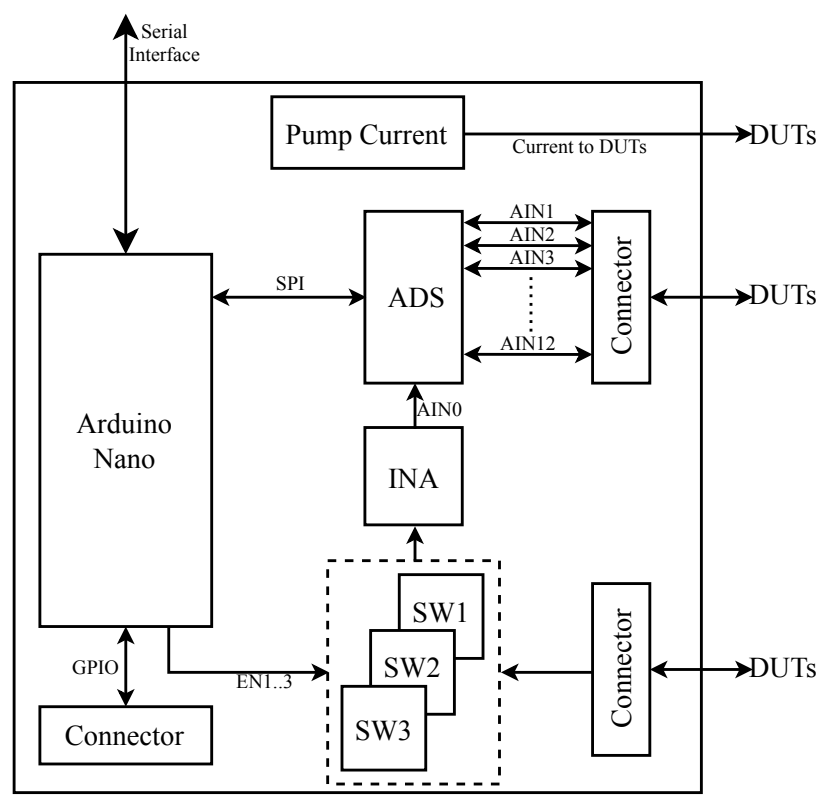

Fig. 5. Acquisition board block diagram.

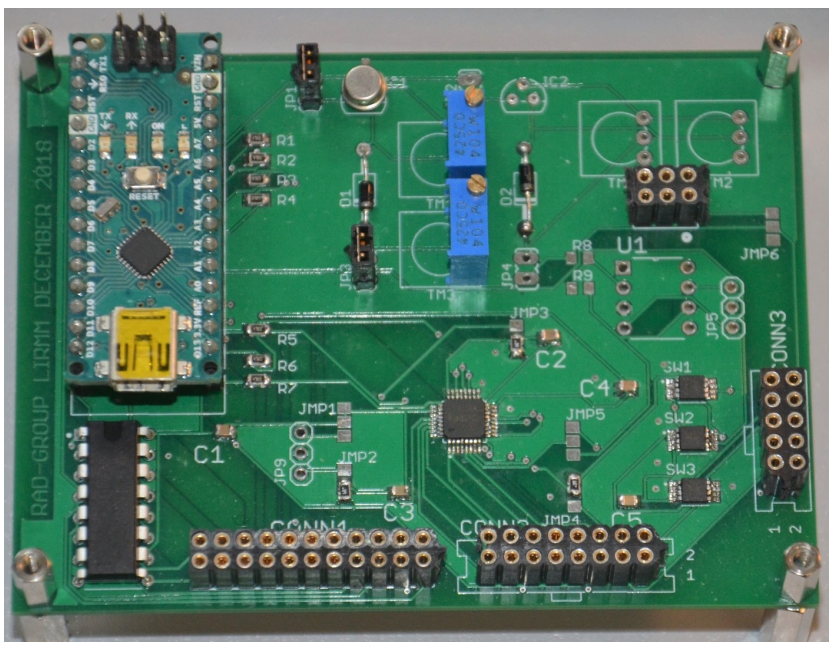

Fig. 6. Acquisition board.

\section{Preliminary Experimental Data}

The RADFET was tested in a radiation environment using a dose rate of $15 \mathrm{rad} / \mathrm{h}$. The $\mathrm{V}_{\mathrm{GS}}$ (th) was measured with accumulated doses from $0.57 \mathrm{krad}$ up to $5.3 \mathrm{krad}$. This range was defined since it is about twice than the calculated one for the mission of the MTCube CubeSat that has been proposed by the authors group and that will last 2 years in a polar orbit of $630 \mathrm{~km} \mathrm{[13].}$

Fig. 7 presents the variation of the threshold voltage versus the absorbed dose. For each TID, the measurement was done using a sampling rate of 5 SPS during a time window of 1 second. The $\Delta V_{G S}($ th) value is the average of these five samples. To analyze the variation response of the sensor, a linear curve fitting was plotted to infer the 
behavior of the variation. The fit curve was plotted following the Eq. 3. where $f(x)$ is the $\Delta \mathrm{V}_{\mathrm{GS}}$ (th) and $x$ is Total lonizing Dose $[\mathrm{krad}(\mathrm{Si})]$.

$$
f(x)=0.03656596 * x+0.02318814
$$

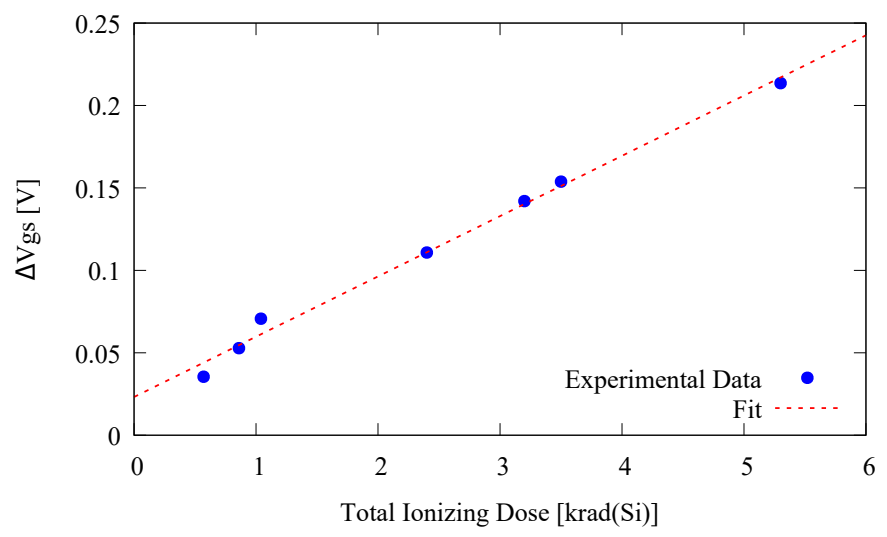

Fig. 7. Threshold shift of a RADFET during irradiation as a function of accumulated radiation dose.

From datasheet components of the same RADFET family, as in [14], the $\Delta \mathrm{V}_{\mathrm{GS}}$ (th) follows an exponential behavior, however, for small ranges of TID, we can consider that it is sufficient linear as shown in Fig. 7 .

\section{CONCLUSION}

In this paper we described a flexible system to interface with a TID sensor. The developed board has the necessary characteristics to acquire the expected data from the RADFET, and also provide a widely voltage range for data acquisition.

Preliminary result presents the expected behavior of the RADFET, since the threshold voltage shifts according the accumulated dose following a linear behavior in the presented TID range.

\section{REFERENCES}

[1] G. Tsiligiannis, "Soft errors in memory devices: Novel methods and practices for dynamic and static testing," Ph.D. dissertation, Université de Montpellier, Montpellier, FR, Sept. 2014.

[2] L. Dilillo, A. Bosser, A. Javanainen, and A. Virtanen, "Space radiation effects in electronics," in Rad-hard Semiconductor Memories, ser. River Publishers Series in Electronic Materials and Devices, J. Nakajima, C. Calligaro, and U. Gatti, Eds., Nov. 2018, pp. 1-64.

[3] J. Wyss, D. Bisello, and D. Pantano, "SIRAD: an irradiation facility at the LNL Tandem accelerator for radiation damage studies on semiconductor detectors and electronic devices and systems," Nuclear Instruments and Methods in Physics Research Section A: Accelerators, Spectrometers, Detectors and Associated Equipment, vol. 462, no. 3, pp. 426-434, Apr. 2001.

[4] M. Bagatin, S. Gerardin, and A. Paccagnella, "lonizing radiation effects in electronic devices with an emphasis on non-volatile memories," in 2015 IEEE International Integrated Reliability Workshop (IIRW). IEEE, oct 2015, pp. 159-159.

[5] N. Rostand, S. Martinie, M. Gaillardin, C. Marcandella, O. Rozeau, J. Lacord, J.-C. Barbe, T. Poiroux, and G. Hubert, "Total lonizing Dose Effects in FDSOI Compact Model for IC Design," IEEE Transactions on Nuclear Science, vol. PP, pp. 1-1, Apr. 2019.

[6] C. Leroy and P.-G. Rancoita, Principles of Radiation Interaction in Matter and Detection, 2nd ed. World Scientific Publishing Company, 2009.

[7] S. Danzeca, J. Cesari, M. Brugger, L. Dusseau, A. Masi, A. Pineda, and G. Spiezia, "Characterization and modeling of a floating gate dosimeter with gamma and protons at various energies," IEEE Transactions on Nuclear Science, vol. 61, no. 6, pp. 3451-3457, 2014.

[8] Tyndall Works, "Transistors, MOSFET, P-Channel (RADFET)," Tyndall RADFET/01 datasheet, 2011.

[9] Texas Instruments, "ADS114S0x Low-Power, Low-Noise, Highly Integrated, 6- and 12-Channel, 4-kSPS, 16-Bit, Delta-Sigma ADC with PGA and Voltage Reference," ADS114S06, ADS114S08 datasheet, Feb. 2017, [Revised Jun. 2017].

[10] ㄴ "3-terminal adjustable current sources," LM134/LM234/LM334 datasheet, Mar. 2000, [Revised May. 2013].

[11] Analog Devices, "1 $\Omega$ Resistance, \pm 15 V/+12 V/ \pm 5 V icmos spst switches," ADG1401, ADG1402 datasheet, Oct. 2009.

[12] Texas Instruments, "INA12x Precision, Low-Power Instrumentation Amplifier," INA128, INA129 datasheet, Oct. 1995, [Revised April. 2019].

[13] V. Gupta, A. L. Bosser, F. Wrobel, F. Saigné, L. Dusseau, A. Mohammadzadeh, and L. Dilillo, "MTCube project: COTS memory SEE groundtest results and in-orbit error rate prediction," in The 4S Symposium, Small Satellites Systems and Services Symposium, Valletta, Malta, 2016.

[14] Tyndall Works, “TY1004 - 400nm RADFET in 8L Side Braze Ceramic Package," TY1004 datasheet, 2015. 